\title{
ESTUDIO DE LA COAGULACIÓN DE PARTÍCULAS COLOIDALES DE AgI POR INFLUENCIA DE QUITOSANO CON SULFATO DE ALUMINIO
}

\author{
Miguel Allcca Chullca ${ }^{\mathrm{a}}$, Nelson Tapia Huananbal ${ }^{*} \mathrm{ab}$, Claudia Villanueva Huerta $^{\mathrm{a}}$, \\ Enrique Guzmán Lezamaa,b, Patricio Muñoz Huillcas ${ }^{\mathrm{a}}$, Neptalí Ale Borja ${ }^{\mathrm{a}}$, \\ Holger Maldonado García ${ }^{a}$
}

\begin{abstract}
RESUMEN
El objetivo del presente trabajo fue determinar las condiciones óptimas de la coagulación de partículas suspendidas de yoduro de plata de carga negativa por la acción de soluciones de quitosano, sulfato de aluminio, así como la acción mixta de ambos coagulantes. La suspensión de partículas coloidales de AgI se obtuvo mediante la mezcla de soluciones diluidas de KI y $\mathrm{AgNO}_{3}$ en exceso de KI. En todos los experimentos el volumen del sistema coloidal fue de $600 \mathrm{~mL}$ y $\mathrm{pH}=6$. Los parámetros óptimos del proceso de coagulación se determinó a base del método de Test de Jarras. Para la solución de quitosano los parámetros óptimos fueron: concentración $0,01 \%, \mathrm{pH}=4,5$ y un volumen de $25 \mathrm{~mL}$; para el sulfato de aluminio: concentración $30 \mathrm{ppm}, \mathrm{pH}=4,0$ y un volumen $25 \mathrm{~mL}$. En el caso de la acción mixta de ambos coagulantes los parámetros óptimos fueron $5 \mathrm{~mL}$ de una solución al $30 \mathrm{ppm}$ de sulfato de aluminio a pH 4,0 y $20 \mathrm{~mL}$ de una solución de quitosano al $0,01 \%$ a $\mathrm{pH}=4,5$. A base de estos parámetros, se estudió la cinética del proceso de coagulación de la suspensión de AgI mediante el método de turbidimetría y se determinó que el proceso de coagulación fue rápido y culminó aproximadamente a los 3 minutos de haber empezado el proceso.
\end{abstract}

Palabras clave: biopolímero, coagulación, floculación, quitosano.

\section{STUDY OF THE COAGULATION OF COLOIDAL PARTICLES OF AgI UNDER THE INFLUENCE OF CHITOSAN WITH ALUMINUM SULFATE}

\begin{abstract}
The objective of the present work was to determine the optimal conditions for the coagulation of suspended particles of silver iodide of negative charge by the action of solutions of chitosan, aluminum sulfate and the mixed action of both coagulants. The suspension of colloidal

a Facultad de Quim. e Ing. Quim., Universidad Nacional Mayor de San Marcos, Lima 1.ntapiah@unmsm.edu.pe b Facultad de Ciencias y Filosofía Alberto Cazorla Talleri. Departamento de Ciencias Exactas-Sección Química,

Universidad Peruana Cayetano Heredia, Lima 31.
\end{abstract}


particles of $\mathrm{AgI}$ was obtained by mixing diluted solutions of $\mathrm{KI}$ and $\mathrm{AgNO}_{3}$ in excess of $\mathrm{KI}$. In all the experiments the volume of the colloidal system was $600 \mathrm{~mL}$ and $\mathrm{pH}=6$. The optimal parameters for the coagulation process were determined based on the Jar Test method. For the chitosan solution the optimal parameters were: concentration $0.01 \%, \mathrm{pH}=4.5$ and a volume of $25 \mathrm{~mL}$; for aluminum sulfate: concentration $30 \mathrm{ppm}, \mathrm{pH}=4.0$ and volume $25 \mathrm{~mL}$. In the case of the mixed action of both coagulants, the optimal parameters were $5 \mathrm{~mL}$ of a $30 \mathrm{ppm}$ solution of aluminum sulfate at $\mathrm{pH} 4.0$ and $20 \mathrm{~mL}$ of a $0.01 \%$ chitosan solution at $\mathrm{pH}=4.5$. Based on these parameters, the kinetics for the coagulation process of the suspension of AgI was studied by means of the turbidimetry method and it was determined that the coagulation process was rapid and ended approximately 3 minutes after beginning of the process.

Key words: biopolymer, coagulation, flocculation, chitosan.

\section{INTRODUCCIÓN}

Las aguas naturales contienen impurezas y partículas supendidas de diversos tamaños de origen mineral u orgánico, que son responsables de la turbidez y color del agua ${ }^{1,2}$. Estos contaminantes causan serios problemas durante el proceso de potabilización del agua 3 .

Para sedimentar las partículas coloidales suspendidas en el agua se usa generalmente el proceso de coagulación y floculación ${ }^{4}$, que consiste en producir la sedimentación de las partículas mediante el uso de diferentes tipos de coagulantes inorgánicos, polímeros naturales y sintéticos. Entre los coagulantes inorgánicos, el más usado es el sulfato de aluminio ${ }^{5}$ por su bajo costo y manejo; sin embargo, después del proceso de floculación quedan trazas del ion de $\mathrm{Al}^{3+}$, las cuales pueden poner en riesgo la salud pública, debido a que este ion favorece el desarrollo del mal de Alzheimer ${ }^{6}$. El proceso de coagulación-floculación produce grandes volúmenes de lodos, que también dan lugar a problemas ambientales ${ }^{7}$, debido al uso de coagulantes inorgánicos y polímeros sintéticos ${ }^{8}$. En este aspecto, el uso de biopolímeros en el proceso de coagulación floculación es de gran importancia para evitar la contaminación con aluminio y polímeros sintéticos no biodegradables ${ }^{9}$.

El quitosano es un polisacárido que en su estructura contiene a grupos aminas y se obtiene a partir de la desacetilación de la quitina. A pH inferior a 7, la mayoría de los grupos amina están protonados y pueden atraer cationes de metales ${ }^{9-11}$ y también pueden unirse a partículas coloidales de carga negativa ${ }^{12-14}$. Esta propiedad se utiliza para producir la coagulación en redes, la cual determina que el quitosano se pueda utilizar como floculante en diferentes aplicaciones, tales como: sedimentación de suspensiones sólidos en la industria de alimentos, separación de microorganismos y colorantes de efluentes textiles ${ }^{15-17}$.

En el presente trabajo se ha determinado los parámetros óptimos del proceso de coagulaciónfloculación de partículas suspendidas de yoduro de plata de carga negativa en agua, producidas por los coagulantes: sulfato de aluminio, quitosano y soluciones mixtas de sulfato de aluminio con quitosano. Utilizando los parámetros óptimos de este proceso de 
coagulación, se ha estudiado también la cinética del proceso de coagulación correspondiente a la acción mixta de estos coagulantes.

\section{PARTE EXPERIMENTAL}

\section{Sistema coloidal de yoduro de plata (AgI)}

Para preparar el sistema coloidal de yoduro de plata por el método de condensación química, se disolvió 3,320 g de AgNO3 (Merck) en 2,4 L de agua destilada en un vaso de 3L de capacidad y en otro vaso se disolvió 1,698 g de KI (Merck) en 1,2 L de agua destilada. Estas soluciones se mezclaron en un agitador magnético (HANNA) durante 20 min.

\section{Solución de quitosano y sulfato de aluminio}

Se pesó $1,0 \mathrm{~g}$ de quitosano de alto peso molecular en polvo (Sigma - Aldrich) y se disolvió en una solución de $100 \mathrm{~mL}$ de ácido acético al $2 \%$ mediante agitación constante con un agitador de paletas (HANNA) a $100 \mathrm{rpm}$ durante $24 \mathrm{~h}$.

Para preparar una solución de $1000 \mathrm{ppm}$ de $\mathrm{Al}^{3+}$, se pesó $12,33 \mathrm{~g}$ de sulfato de aluminio (Merck) y se disolvió en agua destilada en una fiola de un litro.

\section{Determinación de la concentración óptima del quitosano}

De la solución $1 \%(\mathrm{~W} / \mathrm{V})$ de quitosano por dilución se prepararon las siguientes soluciones: $0,004 \% ; 0,008 \% ; 0,01 \% ; 0,015 \% ; 0,02 \%$ y 0,03\% en fiolas de $100 \mathrm{~mL}$. En los vasos del equipo de jarras se colocaron $600 \mathrm{~mL}$ del sistema coloidal, y se ajustó el pH a 6,0 con soluciones $0,1 \mathrm{~N}$ de $\mathrm{NaOH}$ y HCl, utilizando un $\mathrm{pH}$-metro Russell RL060P. A los seis vasos les se agregó sucesivamente $20 \mathrm{~mL}$ de las soluciones de quitosano preparadas anteriormente. Se procedió a agitar las soluciones a $100 \mathrm{rpm}$ durante $3 \mathrm{~min}$, y enseguida a $30 \mathrm{rpm}$ durante 10 minutos, para luego dejarlo reposar durante $20 \mathrm{~min}$, y después se midió la turbidez con un turbidimetro (HANNA - HI 93703). Los resultados se muestran en la fgura 1. De la gráfica que se muestra en esta figura, se deduce que la concentración óptima de quitosano para producir la coagulación es $0,01 \%$ a un $\mathrm{pH}=6$ para este sistema coloidal.

\section{Determinación del volumen óptimo de la solución 0,01 \% de quitosano en la coagulación del sistema coloidal de Agl.}

En los 6 vasos del equipo de jarras se colocaron $600 \mathrm{~mL}$ del sistema coloidal AgI. El pH de cada uno de los vasos se ajustó a 6 con soluciones $0,1 \mathrm{~N}$ de $\mathrm{HCl}$ y $\mathrm{NaOH}$. Luego, a cada uno de los vasos de manera secuencial se agregó 5, 10, 15, 20, 25 y $30 \mathrm{~mL}$ de la solución de quitosano al 0,01\%, y se procedió a agitar en el equipo de jarras a $100 \mathrm{rpm}$ durante $3 \mathrm{~min}$, y después a $30 \mathrm{rpm}$ durante 10 minutos. Se dejaron reposar las muestras durante 20 min e inmediatamente se midió la turbidez, los resultados se muestra en la figura 3.

\section{Determinación del pH óptimo de la solución $0,01 \%$ de quitosano}

Para determinar el efecto del $\mathrm{pH}$ de la solución óptima de quitosano al 0,01\% en el proceso de coagulación y floculación de las partículas coloidales de AgI, se prepararon en 6 fiolas de 
$25 \mathrm{~mL}$ soluciones de quitosano a diferentes $\mathrm{pH}: 3,0 ; 4,0 ; 4,5 ; 5,0 ; 5,5$ y 6,0 . Estas soluciones en forma consecutiva se agregaron a los 6 vasos del equipo de jarras que contenían $600 \mathrm{~mL}$ del sistema coloidal y se procedió a agitar las soluciones a $100 \mathrm{rpm}$ durante 3 min y enseguida a $30 \mathrm{rpm}$ durante 10 minutos, luego se dejó reposar la mezcla obtenida durante 20 min y se midió la turbidez. Los resultados se muestran en la figura 5.

\section{Determinación de la concentración óptima de la solución de sulfato de aluminio}

A partir de la solución de $1000 \mathrm{ppm}$ de $\mathrm{Al}^{3+}$ por dilución en filolas de $100 \mathrm{~mL}$, se prepararon soluciones de 10, 20, 30, 40, 50 y 60 ppm. En los 6 vasos del equipo de jarras se colocaron $600 \mathrm{~mL}$ del sistema coloidal y se ajustó el $\mathrm{pH}$ a 6 . Luego se agregó $20 \mathrm{~mL}$ de cada una de las soluciones de sulfato de aluminio a los 6 vasos mediante agitación constante a 100 rpm durante $3 \mathrm{~min}$, y después a $30 \mathrm{rpm}$ durante $10 \mathrm{~min}$. Se dejaron reposar durante $20 \mathrm{~min}$. Enseguida se tomaron muestras para medir la turbidez. Los resultados se muestran en la gráfica de la turbidez versus la concentración, figura 2. De esta gráfica se deduce que la concentración óptima del $\mathrm{Al}^{3+}$ es 30 ppm.

\section{Determinación del volumen óptimo de la solución 30 ppm de sulfato de aluminio}

Como la concentración óptima de $\mathrm{Al}^{3+}$ para producir la coagulación del sistema coloidal con partículas de AgI de carga negativa es 30 ppm, se preparó 1L de esta solución. En los 6 vasos del equipo de jarras se colocaron $600 \mathrm{~mL}$ del sistema coloidal de AgI y se ajustó el pH a 6,0. En seguida a cada uno de estos vasos en forma sucesiva se agregó 5, 10, 15, 20, 25 y $30 \mathrm{~mL}$ de la solución de sulfato de aluminio y se procedió a agitar las soluciones primero a $100 \mathrm{rpm}$ durante 3 min y después a $30 \mathrm{rpm}$ durante 10 minutos. Se dejaron reposar las soluciones durante 20 min e inmediatamente se midió la turbidez. Los resultados se muestran en figura 4 .

\section{Determinación del pH óptimo de la solución 30 ppm de sulfato de alumnio}

Para determinar el efecto del $\mathrm{pH}$ de la solución $30 \mathrm{ppm} \mathrm{de} \mathrm{Al}^{3+}$ en el proceso de coagulación y floculación de las partículas coloidales de AgI, en 6 fiolas de $25 \mathrm{~mL}$ se prepararon soluciones de 30 ppm de $\mathrm{Al}^{3+}$ a diferentes $\mathrm{pH}: 3,0 ; 4,0 ; 5,0 ; 6,0 ; 7,0$ y 8,0. Estas soluciones, en forma consecutiva, se agregaron a los 6 vasos del equipo de jarras que contenían $600 \mathrm{~mL}$ del sistema coloidal, y se procedió a agitar las soluciones a $100 \mathrm{rpm}$ durante $3 \mathrm{~min}$, y enseguida a $30 \mathrm{rpm}$ durante 10 minutos. Luego se dejó reposar la mezcla obtenida durante 20 min, y después se midió la turbidez. Los resultados se muestran en la figura 6.

\section{Determinación de la proporción óptima entre el volumen de quitosano y sulfato de aluminio en el proceso de coagulación}

Con la finalidad de determinar esta proporción, en los 6 vasos del equipo de jarras se colocaron $600 \mathrm{~mL}$ del sistema coloidal de AgI y se ajustó su pH a 6,0. En seguida se agregó $2,5,5,10,15,20$ y 22,5 mL en forma secuencial de la solución de sulfato de aluminio al 30 ppm y $\mathrm{pH}=4,0$. La mezcla obtenida se agitó durante 3 min a $30 \mathrm{rpm}$; luego se adicionó 22,5, $20,15,10,5$ y $2,5 \mathrm{~mL}$ de la solución $0,01 \%$ de quitosano a un $\mathrm{pH}=4,5$, y se procedió agitar la mezcla a 100 rpm durante 3 minutos, y después a 30 rpm durante 10 minutos. Se dejó reposar por 20 minutos y se midió la turbidez. Los resultados se muestran en figura 7. 


\section{Cinética de la coagulación del sistema coloidal de AgI}

Para estudiar la cinética del proceso de coagulación, se colocó $600 \mathrm{~mL}$ del sistema coloidal de AgI en un vaso de 1L. En seguida se adicionó $5 \mathrm{~mL}$ de la solución de Al3+ de $30 \mathrm{ppm}$ con un $\mathrm{pH}=4,0$, agitando la mezcla a $30 \mathrm{rpm}$ durante 3 min e inmediatamente se adicionó $20 \mathrm{~mL}$ de quitosano al $0,04 \%$ a $\mathrm{pH}=4,5$ y se procedió a agitar a $100 \mathrm{rpm}$ por 3 minutos, y finalmente a $30 \mathrm{rpm}$ por 10 minutos. Luego, se extrajeron periódicamente muestras de $20 \mathrm{~mL}$ de esta mezcla para determinar la turbidez. Los resultados se muestran en la figura 8.

\section{RESULTADOS Y DISCUSIÓN}

\section{Proceso de coagulación en función de la concentración de quitosano y sulfato de aluminio} El quitosano es un polisacárido que se encuentran en gran abundancia en la naturaleza. En su estructura molecular contiene una gran cantidad de grupos amino $\left(-\mathrm{NH}_{2}\right)$ y grupos hidroxilos $(-\mathrm{OH})$, los cuales poseen un par de electrones libres, que se utilizan para formar quelatos con los iones de metales divalentes. Además, el grupo amino del quitosano puede ser protonado en una solución acuosa ácida, y de esta manera se transforma en un poliectrólito catiónico. Esta propiedad se utiliza para producir la coagulación de partículas de carga negativa y moléculas de colorantes aniónicos de la industria textil ${ }^{14-17}$. Por esta razón se ha utilizado el quitosano en la coagulación de partículas coloidales de carga negativa de AgI. En la figura 1 se muestra la gráfica de datos experimentales de la turbidez en función de la concentración de quitosano, determinada mediante la técnica de prueba de jarras (test de jarras). De esta gráfica se deduce que la concentración óptima para producir la coagulación de las partículas suspendidas de carga negativa de AgI es $0,01 \%$ a un $\mathrm{pH}=6$.

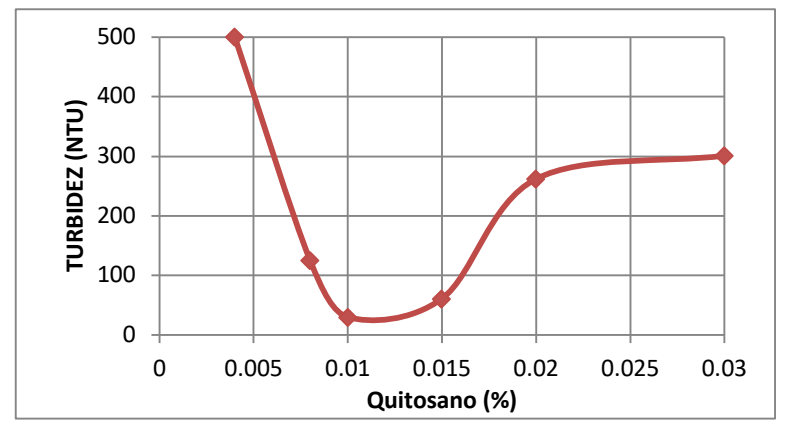

Figura 1. Turbidez del sistema coloidal de Agl en función de la concentración del quitosano.

En la figura 2 se muestra la gráfica de la turbidez del sistema coloidal de AgI, que se encontraba a un $\mathrm{pH}=6$, en función de la concentración de sulfato de aluminio. De esta gráfica se deduce que la concentración óptima de sulfato de aluminio para producir la coagulación y floculación del sistema coloidal AgI es 30 ppm. 


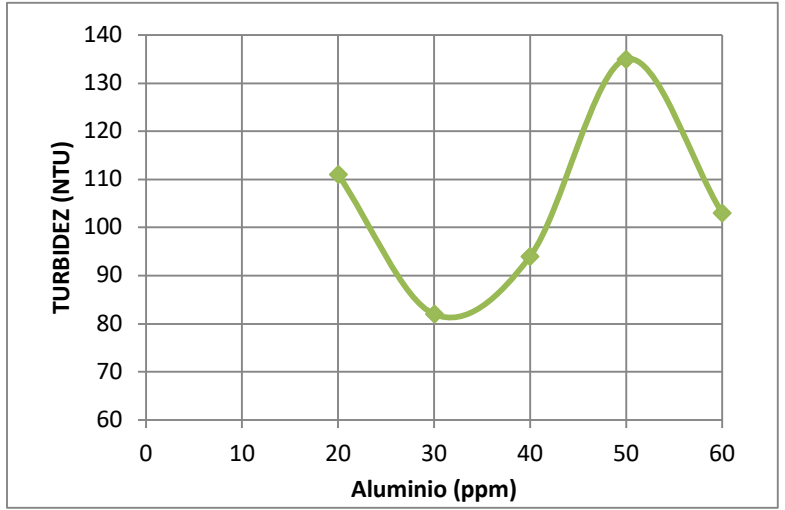

Figura 2. Turbidez del sistema coloidal de Agl en función de la concentración de $\mathrm{Al}^{3+}$

\section{Coagulación en función del volumen de quitosano y sulfato de aluminio}

Una vez que se ha determinado la concentración óptima de la solución coagulante, es también muy importante determinar el volumen óptimo de esta solución que produce la coagulación de $600 \mathrm{~mL}$ del sistema coloidal. En las figura 3 y 4 se muestran los resultados experimentales obtenidos de la turbidez del sistema coloidal en función del volumen de la solución óptima de quitosano $(0,01 \%)$ y de la solución 30 ppm de sulfato de aluminio. En ambas gráficas se tiene dos mínimos locales, sin embargo los segundos mínimos dan un valor muy pequeño de la turbidez, que nos indica que prácticamente se han removido un $95 \%$ de las partículas coloidales de AgI. Por consiguiente el volumen óptimo de la solución coagulante para ambos casos es aproximadamente de $25 \mathrm{~mL}$.

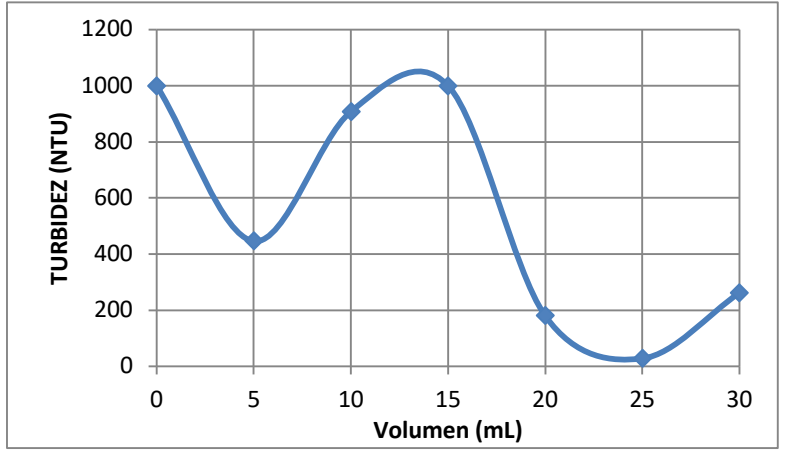

Figura 3. Turbidez del sistema coloidal de Agl en función del volumen de la solución $0,01 \%$ de quitosano a $\mathrm{pH}=4$. 


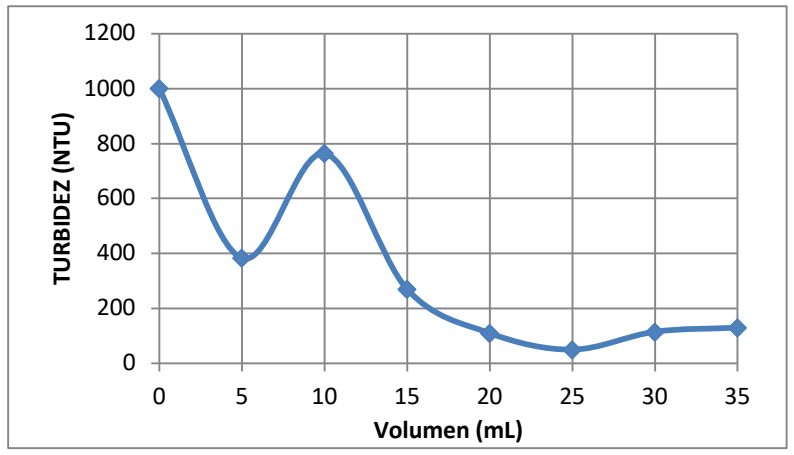

Figura 4. Turbidez del sistema coloidal AgI en función del volumen de la solución $30 \mathrm{ppm}$ de sulfato de aluminio a $\mathrm{pH}=4,5$

\section{Coagulación del sistema coloidal de AgI en función del pH de la solución de quitosano y sulfato de aluminio}

La molécula de quitosano tiene en sus estructura una gran cantidad de grupos hidroxilo $(-\mathrm{OH})$ y grupos amino $\left(-\mathrm{NH}_{2}\right)$, los cuales tienen un par de electrones que pueden donar para formar complejos con los cationes de metales. Por esta razón en soluciones acuosas casi neutras se utiliza para remover cationes de metales pesados. A pH el grupo amino del quitosano se protona $\left({ }^{-} \mathrm{NH}_{3}\right)$ transformándose en un polielectrólito de carga positiva18. Esta propiedad se utiliza para producir la coagulación de colorantes catiónicos y partículas coloidales de carga positiva. En el caso de la solución de sulfato de aluminio el ion coagulante es el $\mathrm{Al}^{3+}$, el cual fácilmente neutraliza a la doble capa eléctrica de las partículas coloidales de AgI. Su capacidad de coagulación también depende del $\mathrm{pH}$ de la solución de sulfato de aluminio y del $\mathrm{pH}$ del sistema coloidal. Por esta razón se estudió la influencia del $\mathrm{pH}$ de las soluciones óptimas de quitosano $(0,01 \%, \mathrm{~V}=25 \mathrm{~mL})$ y sulfato de aluminio $(30 \mathrm{ppm}$ y $\mathrm{V}=25 \mathrm{~mL})$ en la coagulación de $600 \mathrm{~mL}$ del sistema coloidal, que se encontraba a un $\mathrm{pH}$ 6. Los resultados de este estudio se reportan en las gráficas de la turbidez versus el $\mathrm{pH}$ que se muestran en las figuras 5 y 6 . De estas gráficas se deduce que el pH óptimo de la solución $0,01 \%$ de quitosano es 4,5 y para la solución 30 ppm de sulfato de aluminio es 4 , alcanzándose una remoción de partículas coloidales de $97 \%$. 


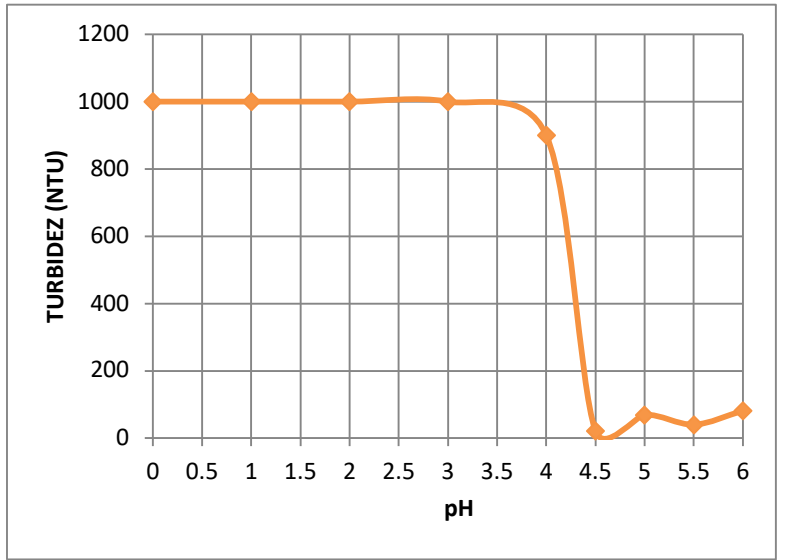

Figura 5. Efecto del $\mathrm{pH}$ de quitosano sobre la turbidez.

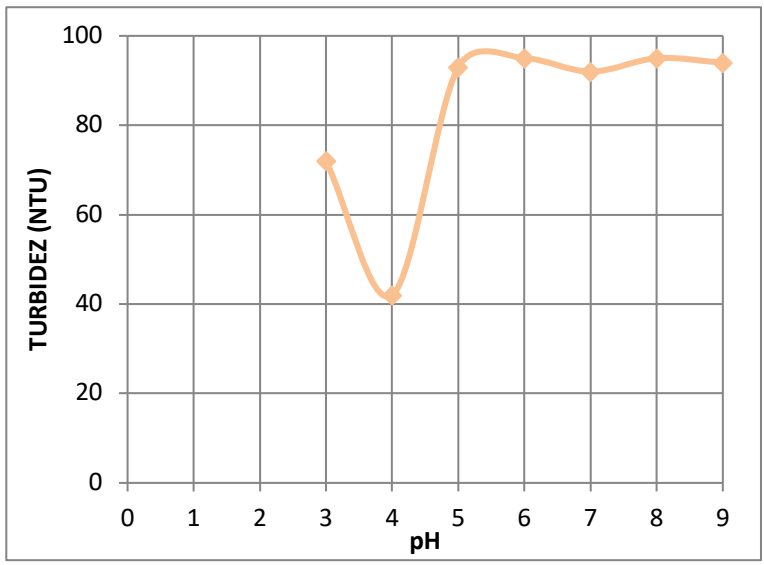

Figura 6. Efecto del pH de aluminio sobre la turbidez.

\section{Proporción óptima del volumen de quitosano y sulfato de aluminio en la coagulación del sistema coloidal de AgI}

En los pasos anteriores se han determinado los parámetros óptimos de las soluciones coagulantes de quitosano (concentración $0,01 \%$, volumen $25 \mathrm{~mL}$ y $\mathrm{pH}=4,5$ ) y sulfato de

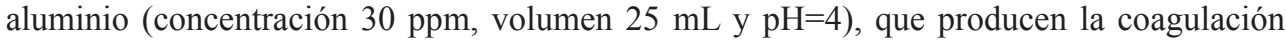
y floculación de $600 \mathrm{~mL}$ del sistema coloidal de AgI . Teniendo esta información se puede examinar la acción mixta del quitosano y sulfato de aluminio en la coagulación del sistema coloidal, los resultados de esta acción mixta se muestran en la figura 7. De la gráfica de la turbidez versus el volúmen de cada solución coagulante, que se ha agregado simultáneamente al sistema coloidal, se deduce que la proporción óptima de cada coagulante es $5 \mathrm{~mL}$ de sulfato de aluminio y $20 \mathrm{~mL}$ de quitosano. En este caso se alcanza el 98,2 \% de remoción las partículas suspendidas de yoduro de plata. 


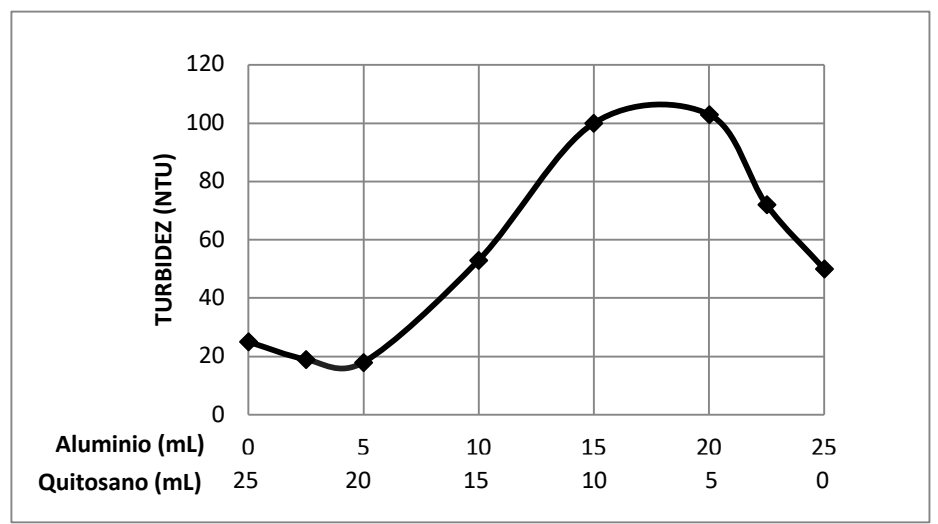

Figura 7. Turbidez del sistema coloidal en función de la proporción en volumen de Quitosano - Aluminio (Aluminio 30 ppm - Quitosano 0,01\%)

\section{Cinética de la coagulación del sistema coloidal de AgI por la acción mixta de sulfato de aluminio y quitosano}

El estudio de la cinética del proceso de coagulación y floculación permite determinar el tiempo óptimo en que se produce la separación de las partículas coloidales suspendidas por acción de los coagulantes y floculantes. En el presente caso, como la partícula coloidal tiene carga negativa, entonces el ion que produce la neutralización de la doble capa eléctrica es el $\mathrm{Al}^{3+}$. Una vez que las partículas son neutralizadas, desaparece la repulsión electroestática entre ellas $\mathrm{y}$, por consiguiente, se unen fácilmente para dar origen a la formación de coágulos de pequeñas dimensiones al inicio del proceso, y después estos coágulos se unen en flóculos que, por acción de la fuerza de gravedad precipitan. Para acelerar el proceso de floculación del sistema coloidal de AgI se ha utilizado el quitosano. Este biopolímero a través de grupos aminos protonados a bajo $\mathrm{pH}$ se une a las partículas coloidales de carga negativa y a los coágulos formados, dando lugar a la formación de flóculos muy pesados que precipitan rápidamente. De la gráfica de la tubidez en función del tiempo de coagulación - floculación, que se muestra en la figura 8 , se deduce que la acción mixta del coagulante $\mathrm{Al}^{3+} \mathrm{y}$ del floculante quitosano es muy rápida, pues culmina a los $3 \mathrm{~min}$ de haber empezado el proceso de coagulación-floculación. 


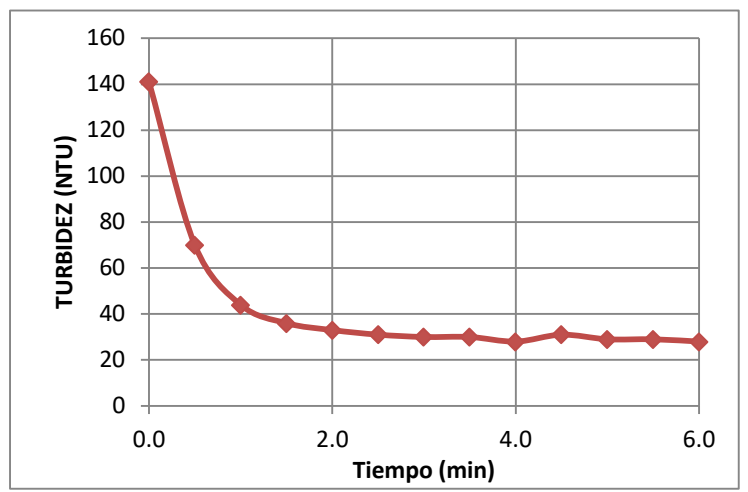

Figura 8. Curva de cinética de coagulación

\section{CONCLUSIONES}

Mediante el uso del método de Test de Jarras y turbidimetría se ha determinado que para producir la coagulación - floculación del sistema coloidal de AgI, $600 \mathrm{~mL}$ a pH 6, por acción de una solución de quitosano, los parámetros óptimos son: concentración $0,01 \%$, volumen óptimo $25 \mathrm{~mL}$ y pH 4,0; para el sulfato de aluminio se tiene: concentración 30 ppm, pH 4,5 y volumen $25 \mathrm{~mL}$; para la acción mixta de ambos coagulantes, considerando al sulfato de aluminio como coagulate y al quitosano como floculante se tiene: volumen de quitosano $20 \mathrm{~mL}$ y volumen de sulfato de aluminio $5 \mathrm{~mL}$, siendo la remoción de partículas coloidales del $98 \%$. Del estudio de la cinética de coagulación - floculación producido por ambos coagulantes en el sistema coloidal de AgI, se ha determinado que este proceso es rápido y termina a los $3 \mathrm{~min}$.

\section{AGRADECIMIENTO}

Los autores reconocen y agradecen al Vicerectorado de Investigación y Posgrado de la UNMSM por el soporte financiero al proyecto C17071771, (2017) "Investigación de la separación selectiva de iones de metales pesados y colorantes de la industria textil de disolución acuosa por procesos de adsorción y coagulación con biopolímeros" 


\section{REFERENCIAS BIBLIOGRÁFICAS}

1. Romero J. Calidad del agua. México D.F.: Editorial Alfaomega; 2000.

2. Castillo J, Bracho N, Vargas L, Romeron y Aldana G. Coagulant selection for water treatment process in plant "C" Maracaibo. Rev Téc Fac Ing Univ del Zulia. 2000; 23(1): $169-178$.

3. Franceschi M, Girou A, Carro-Diaz A, Maurette M, Pueech-Costes E. Optimisation of the coagulation-flocculation process of raw water by optimal design method. Water Res. 2002; 36(1): 3561-3575.

4. Lárez C. Algunos usos del quitosano en sistemas acuosos. Rev Iberoam Polím. 2003; 4(1): 91- 109.

5. Jiang J-Q, Lloyd B. Progress in the development and use of ferrate (VI) salt as an oxidant and coagulant for water and waste water treatment. Water Res. 2002; 36(1): 1397-1408.

6. McLachlan DRC. Aluminium and the risk for Alzheimer's disease. Environmetrics. 1995; (6): 233-275.

7. Renaut F, Sancey B, Badot P, Crini G. Chitosan for coagulation/flocculation processesAn eco-friendly approach. Eur Polym J. 2009; 45(1): 1337-1348.

8. Fuentes L, Contreras W, Perozo R, Mendoza I, Villegas Z. Uso del quitosano de Litopenaeus Schmitti (Decapoda, Penaeidae) en el tratamiento de agua para consumo humano. Multiciencias. 2008; (8): 281-287.

9. Guibal E, Roussy J. Coagulation and flocculation of solutions containing dye using a biopolymer (Chitosan). React Funct Polym. 2007; 67(1): 33-42.

10. Guzman J, Saucedo I, Navarro R, Revilla J, Guibal E. Vanadium Interactions with chitosan: influence of polymer protonation and metal speciation. Langmuir. 2002; 18(5): 1567-173.

11. Yoshida H, Okamoto A, Yamasaki H, Kataoka T. Breakthrough curve for adsorption of acid dye on crosslinked chitosan fiber. Stud Surf Sci Catal. 1993; 80:767-774.

12. Chi F, Cheng W. Use de Chitosan as coagulant to treat wasterwater from milk processing plant. J Polym Environ. 2006; 14(2): 411-417.

13. Strand PS, Varum KM, Stgaard K. Interactions between chitosans and bacterial Suspensions: adsorption and flocculation. Coll Surf B. 2003; (27): 71-81.

14. Hassan M, Li T, Noor Z. Coagulation and flocculation treatment of wastewater in textile industry using chitosan. JCEIB. 2009; 4(1): 43-53.

15. Domar A, Rinaudo M, Terrasin C. Adsorption of chitosan and a quarternized derivate on kaolinite. J Appl Polym Sci. 1989; (38): 1799-1806.

16. Divakaran R, Pillai VNS. Flocculation of river silt using chitosan. Water Res. 2002; (36): 2412-2418.

17. Hassiba Z, Madani D, Amna S, Hakim L, Nabil M. Coagulation flocculation Test of Keddara's water dam using chitosan and sulfate Aluminium. Procedia Eng. 2012; (33): 254-260.

18. Zeng D, Wu J, Kennedy JF. Application of a chitosan flocculant to water treatment. Carbohydr Polym. 2008; (71): 135 - 139. 